\title{
Towards Whole Brain Segmentation by a Hybrid Model
}

\author{
Zhuowen Tu and Arthur W. Toga \\ Lab of Neuro Imaging, School of Medicine \\ University of California, Los Angeles, USA
}

\begin{abstract}
Segmenting cortical and sub-cortical structures from 3D brain images is of significant practical importance. However, various anatomical structures have similar intensity patterns in MRI, and the automatic segmentation of them is a challenging task. In this paper, we present a new brain segmentation algorithm using a hybrid model. (1) A multiclass classifier, PBT.M2, is proposed for learning/computing multi-class discriminative models. The PBT.M2 handles multi-class patterns more easily than the original probabilistic boosting tree (PBT) 11, and it facilitates the process, eventually, toward whole brain segmentation. (2) We use an edge field, by learning, to constraint the region boundaries. We show the improvements due to the two new aspects both numerically and visually, and also compare the results with those by FreeSurfer [2]. Our algorithm is general and easy to use, and the results obtained are encouraging.
\end{abstract}

\section{Introduction}

Segmenting cortical and sub-cortical structures from 3D brain images is a very important task. There has been considerable recent work on 3D segmentation in medical imaging and we cite several representative ones due to the space limit 132 29. The problem is usually tackled in a MAP (maximize a posterior) framework in which appearance models are defined and shape priors used to capture underlying shape regularity. Existing approaches can be roughly categorized into two groups: (1) one which puts strong efforts into the shape priors [139]; (2) and the other which classifies pixels/voxels using various features 6 6 8 .

In [1, a hybrid model for brain anatomical structure segmentation was presented. The system [1] adopted a PBT [11] multi-class classifier to select and combine hundreds of cues such as intensity, gradients, curvatures, and locations to model ambiguous appearance patterns locally. However, the PBT multi-class classifier performs 2-way split only, and it is not efficient to use in performing whole brain segmentation, in which there are many cortical and sub-cortical structures.

In this paper, we present a new brain segmentation algorithm using a hybrid model. (1) A multi-class classifier, PBT.M2, is proposed for learning/computing multi-class discriminative models. The PBT.M2 handles the multi-class patterns more easily than PBT [11, and it facilitates the process, eventually, toward whole 
brain segmentation. (2) We use a learned edge field to constrain the structure boundaries. The edges are also learned and computed by fusing many local photometric and geometric features across different scales.

\section{Hybrid Discriminative/Generative Model}

We give the basic problem formulation in this section. Brain imaging mostly deals with 3D images, which are referred to as volumes for the rest of the paper. For an input volume, $\mathbf{V}$, the task of brain segmentation is to obtain the full partition of each anatomical structure of interest. A solution $W$ can be denoted as $W=\left\{R_{k}, k=0, . ., T\right\}$, where $R_{0}$ is the background region, and $R_{k}, k=1, . ., T$ denote the anatomical structures. We have $\bigcup_{k=0}^{T} R_{k}=\Lambda$ where $\Lambda$ defines the 3D lattice of the input $\mathbf{V} . R_{i} \cap R_{j}=\emptyset, \forall i \neq j$. Let the optimal solution $W^{*}$ to be the one which minimizes an energy

$$
E(W, \mathbf{V})=E_{A P}(W, \mathbf{V})+\alpha_{1} E_{e d g}(W, \mathbf{V})+\alpha_{2} E_{P C A}(W)+\alpha_{3} E_{S M}(W) .
$$

The first term, $E_{A P}(W, \mathbf{V})$, corresponds to the discriminative model $p(y \mid \mathbf{V}(N(s))$ modeling the joint appearances

$$
E_{A P}(W, \mathbf{V})=-\sum_{k=0}^{T} \sum_{s \in R_{k}} \log p(y=k \mid \mathbf{V}(N(s))),
$$

where $N(s)$ includes all the voxels in the sub-volume, and $y \in\{0, \ldots, T\}$ is the label/class for each voxel. $p(y=k \mid \mathbf{V}(N(s))$ essentially computes the classification probability of voxel $s$ belonging to structure $k$.

$E_{e d g}(W, \mathbf{V})$, on the other hand, focuses on the regions boundary and

$$
E_{\text {edg }}(W, \mathbf{V})=-\sum_{k=0}^{T} \sum_{s \in \partial R_{k}} \log p(E G(s)=\text { on } \mid \mathbf{V}(N(s)))
$$

$\partial R_{k}$ denotes the surface of $R_{k}$ and $p(E G(s)=$ on $\mid \mathbf{V}(N(s)))$ computes the probability of voxel $s$ being on the boundary of a region. The discriminative model, $E_{A P}(W, \mathbf{V})$, captures complex appearances as well as the local geometry by looking at a sub-volume. It also provides context information. If a very accurate $E_{A P}(W, \mathbf{V})$ can be learned, then $E_{e d g}(W, \mathbf{V})$ is not needed. However, due to the large intra-class variability, it is often hard to perfectly classify all the voxels. $E_{\text {edg }}(W, \mathbf{V})$ is more robust against global intensity pattern change than $E_{A P}(W, \mathbf{V}) . E_{P C A}(W)$ and $E_{S M}(W)$ represent the generative models about the shape prior $p(R)$. $E_{P C A}(W)$ is defined on the global shape model of each structure and $E_{S M}(W)$ encourages the region boundaries to be smooth. $\alpha_{1}, \alpha_{2}$, and $\alpha_{3}$ are the weights balancing between how much we rely on the discriminative model, the global shape regularity, and the local smoothness. These weights are learned automatically and the details can be found in [1]. 


\section{Learning Discriminative and Generative Models}

This section gives more details about how the discriminative and generative models are learned and computed.

\subsection{Learning Discriminative Models}

To compute $E_{A P}$, our task is to learn and compute the discriminative model $p(y=k \mid \mathbf{V}(N(s)))$. Each input sample is a sub-volume and the output is the probability of the center voxel $s$ being on region $R_{k}, k=0 . . T$. This is not an easy task due to the complex appearance patterns of $\mathbf{V}(N(s))$. [1 adopted a probabilistic boosting tree (PBT) approach to learn and compute a multi-class classifier. However, the original PBT performs 2-way split only, which is not always efficient. For example, one has to compute at least two strong classifiers in order to classify 4 different classes. On the other hand, it is desirable to do 2-way split when there are many available classes since learning a single node classifier to perform, say, 25-class classification is both difficult and time-consuming. Let the training set be $S=\left\{\left(\mathbf{V}_{a}, y_{a}\right), a=1 . . n\right\}$ where $\mathbf{V}_{a}$ is a $11 \times 11 \times 11$ volume sample, $y_{a} \in\{0 . . T\}$ denotes its class label, and $n$ is the total sample number. Let $p(S, j)$ be the proportion of samples in $S$ that belong to the $j$ th class. The entropy of set $S$ can be defined as $\operatorname{Info}(S)=-\sum_{j=0}^{T} p(S, j) \log _{2}(p(S, j))$.
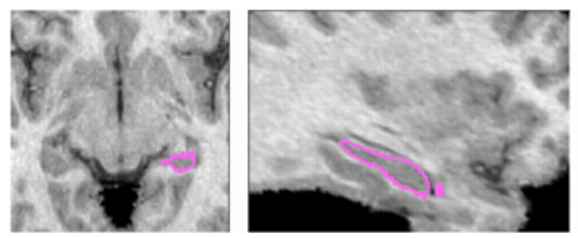

manual labels
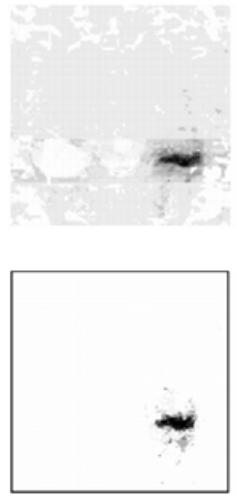

by PBT

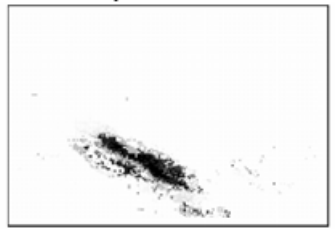

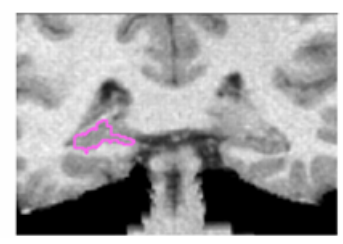
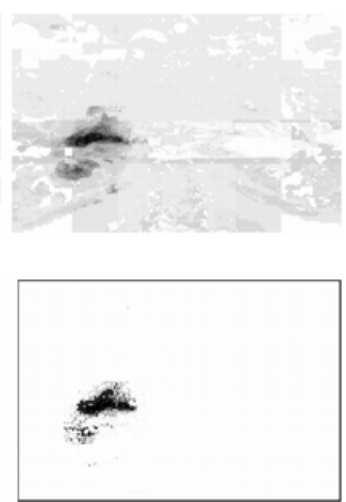

by PBT.M2

Fig. 1. Classification results of a volume on left hippocampus. The first row shows three slices of part of a volume with the left hippocampus highlighted. The three figures in the second row display the soft map, $p(y=1 \mid \mathbf{V}(N(s)))$ (left hippocampus) at three typical slices by the original PBT algorithm, and the third row shows the result by the PBT.M2. 
Apparently, the smaller the entropy is, the sparser the classes are in the set. We want to recursively construct a decision tree in which each tree node is either an AdaBoost [3] (2-class) strong classifier or AdaBoost.MH [4] (m-class) strong classifier. For a strong classifier $H \in\left\{H_{2}, H_{m}\right\}$ (either 2-class or m-class), it splits $S$ into $t$ sub-groups $\left(S_{1}, \ldots, S_{t}\right)$. We choose the $H$ which obtains the biggest information gain

$$
G(S, H)=-\sum_{i=1}^{t} \frac{\left|S_{i}\right|}{|S|} \operatorname{Info}\left(S_{i}\right)-\operatorname{cost}(H),
$$

where the first term is similar to that in the well-known $\mathrm{C} 4.5$ algorithm [10] and $\operatorname{cost}(H)$ computes the total computational cost for strong classifier $H$. Therefore, the choice of $H$ balances between how well to separate the current set and the computational cost. Fig. (2) outlines the basic PBT.M2 algorithm.

Given: Labeled training examples $S=\left\{\left(\mathbf{V}_{a}, y_{a}\right), a=1 . . n\right\}$ with each $y_{a} \in\{0 . . T\}$

- Train a 2-class AdaBoost 3 classifier, $H_{2}$, and m-class $(\mathrm{m}=\mathrm{T}+1)$ AdaBoost.MH 4] classifier, $H_{m}$.

- Choose the strong classifier, $H \in\left\{H_{2}, H_{m}\right\}$, which maximizes the information gain $G(S, H)$.

- Split the training set $S$ using $H$ and recursively train a sub-tree.

- Stop the tree node expansion if the error is smaller than a threshold.

Fig. 2. PBT.M2 algorithm

PBT.M2 learns and computes an overall multi-class discriminative probability, like PBT [11, by

$$
p(y \mid \mathbf{V})=\sum_{l_{1}} \tilde{p}\left(y \mid l_{1}, \mathbf{V}\right) q\left(l_{1} \mid \mathbf{V}\right)=\sum_{l_{1}, . ., l_{n}} \tilde{p}\left(y \mid l_{n}, \ldots, l_{1}\right), \ldots, q\left(l_{2} \mid l_{1}, \mathbf{V}\right) q\left(l_{1} \mid \mathbf{V}\right),
$$

where $l_{i}$ represents the $i$ th layer in the tree, and $q\left(l_{i}\right)$ computes the discriminative probability by each boosting node in the tree. Fig. (1) illustrates an abstract version of the PBT.M2.

The key of the PBT.M2 classifier is that it is capable of hierarchically fusing a set of informative features automatically selected from a large pool of candidate features $(5,000)$. These features carry both intensity and local geometric properties of each voxel in a sub-volume. They include the $x, y, z$ coordinates of the position of each voxel of interest, intensity value, gradients, curvatures, and various 3D Haar features computed in the sub-volume. Fig. (10) shows the classification results by PBT and PBT.M2, and we can clearly see the improvement.

In our experiments, some features selected by the PBT.M2 are: (1) coordinate $\mathrm{x}$ of the center voxel $s$; (2) Haar filter of size $9 \times 7 \times 7$; (3) gradient at $s$;

\section{Learning $E_{\text {edg }}(W, \mathbf{V})$}

To further constrain the region boundary, we use an explicit boundary term $E_{e d g}(W, \mathbf{V})$. Existing work using explicitly edge terms [5] require to specify parameters, e.g. scale, and they are not adaptive. We use PBT to learn boundary 
voxels and the learning/computing process is nearly the same as that in learning the voxel label classification. The only difference is that their training annotations are different. In Fig. (5) we show how the results are affected by using the learned edge field.

\subsection{Learning Generative Shape Models}

In this paper, a simple PCA model, similar to [131], is adopted based on the signed distance function of the shape. Training images are registered first and we align all the anatomical structures according to their centers when learning the shape prior. For each manually labeled anatomical structure, e.g. left hippocampus $R_{1}$, its corresponding signed distance map (SDM) $S_{1}$ is computed in which the value of each voxel $s$ represents its distance to the surface. We can learn a PCA model by $S=U \beta+\bar{S}, \quad Q=U \Sigma V^{T}$, where $\bar{S}$ is the mean of the SDM for the training shapes, and $Q$ is a matrix with each row vector being a training sample $S_{i}-\bar{S}$. The third energy in eqn. (11) becomes

$$
E_{P C A}=\sum_{k=1}^{T} \frac{1}{2} \beta_{k}^{T} \Sigma_{k} \beta_{k}+\alpha_{4}\left\|U_{k}^{T} U_{k}\left(S_{k}-\bar{S}_{k}\right)\right\|_{2} .
$$

Another energy term, $E_{S M}=\sum_{k=0}^{T} \int_{\partial R_{k}} d A$, is added to encourage smooth surfaces. $\int_{\partial R_{k}} d A$ is the area of the surface of region $R_{k}$. When the total energy is being minimized in a variational approach, this term corresponds to the force that encourages each boundary point to have small mean curvature, resulting in smooth surfaces.

\section{Segmenting 3D Brain Volumes}

The goal of the segmenting stage is to find the optimal segmentation/solution which minimizes the energy in eqn. (1). In our problem, the number of anatomical structures and their approximate positions are known. Therefore, we can apply a PDE approach to perform energy minimization. We use steepest descent to minimize energy $E(W, \mathbf{V})$ in eqn. (11). We can derive the motion equations for $E_{A P}$, $E_{e d g}, E_{P C A}$, and $E_{S M}$ similarly as in [1] (see the details in the supplementary document).

\subsection{The Outline of the Algorithm}

Our algorithm is summarized in this section.

Training: (1) For a set of training volumes with the anatomical structures manually delineated, we train a PBT.M2 to learn the discriminative model $p(y \mid \mathbf{V}(N(s)))$. (2) For a set of training volumes with the annotated boundary voxels, train a PBT to learn the discriminative model for the edges. (3) For a set 
of training shapes for each anatomical structure, we learn its PCA shape model as discussed in sect. 3.2. (4) Learn $\alpha_{1}, \alpha_{2}$ and $\alpha_{3}$ to combine the discriminative and generative models.

Testing: (1) Given an input volume $\mathbf{V}$, we compute $p(y \mid \mathbf{V}(N(s)))$ for each voxel and assign it with the label of the highest probability, to obtain a classification map. (2) Based on the classification map, obtain an initial segmentation in which all the anatomical structures are topologically connected. (3) Perform boundary evolution in minimizing the total energy $E$ shown in eqn. (11). (4) Stop the algorithm after several iterations.

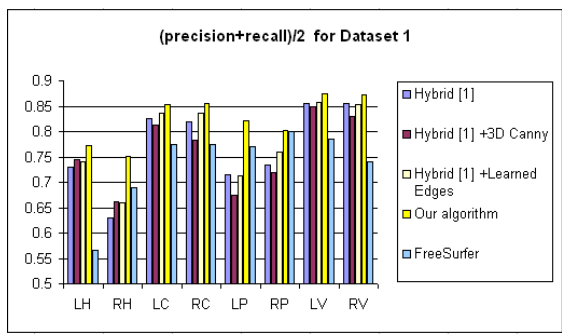

(a) 8 structures on 15 testing volumes

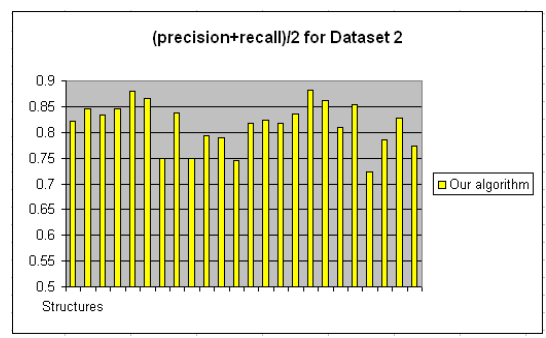

(b) 25 structures on 25 testing volumes.

Fig. 3. Error measures on the testing volumes of the two datasets. The new algorithm obtains the best results which illustrate the effectiveness of using PBT.M2 and learned edge field (see text for more explanation). Fig. (5) visually shows the comparison on a testing volume for dataset 1 . Fig. (4) shows the result on a volume in dataset 2 . The 25 structures include: hippocampus, putamen, caudate, superior temp., Superior occipital, middle frontal...

\section{Experiments}

High-resolution 3D SPGR T1-weighted MR images were acquired on a GE Signa $1.5 \mathrm{~T}$ scanner. All the volumes shown in this paper are registered by 12 . For the first dataset of 28 volumes annotated by neuroanatomists for 8 sub-cortical structures. 14 volumes are randomly selected for training and the rest 14 are used for testing. We apply the algorithm stated in sect. 4.1 to segment the eight anatomical structures on both the training and testing volumes. The training and testing processes are repeated twice and we observe the same performances. For the second dataset of 40 volumes with 25 sub-cortical and cortical structures are manually delineated, 15 volumes were used for training and 25 are used for testing.

To quantitatively measure the effectiveness of our algorithm, errors are measured using several criteria, and they appear to be all consistent. We report the precision and recall measure here. Let $R$ be the set of voxels annotated by an expert and $\hat{R}$ be the voxels segmented by the algorithm. The precision and recall are 
measured as Precision $=\frac{R \cap \hat{R}}{\hat{R}}$, and Recall $=\frac{R \cap \hat{R}}{R}$. The average is (Precision + Recall)/2. Fig. (4) shows the error measured on the two datasets. To test the effectiveness of using learned edge field and PBT.M2, we conducted several experiments: (1) the original hybrid model [1]; (2) hybrid model in [1]+3D Canny edges; (3) hybrid model in $11+$ learned edges; (4) the overall model reported in this paper. It is observed that the new algorithm performs the best and the model using learned edges outperforms the one with $3 \mathrm{D}$ Canny edges [7]. To directly
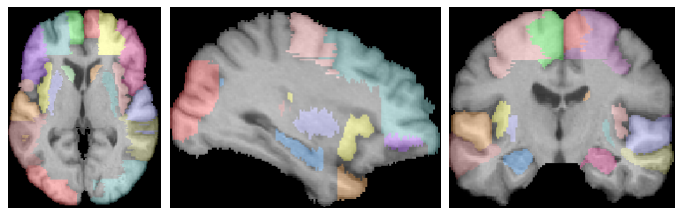

manul delineations
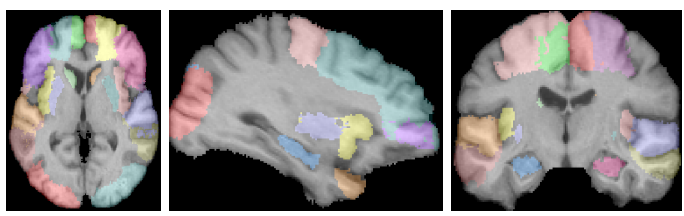

by our algorithm

Fig. 4. Results on a typical testing volume with 25 cortical and sub-cortical structures delineated. The second row shows the result by the algorithm reported in this paper. We are in the process of getting more $(50 \sim 100)$ structures manually annotated for training our algorithm. compare our algorithm to an existing state of art algorithm, we tested the MRI data using FreeSurfer [2] and our results are better than theirs. Fig. (5) visually shows the comparison in different trials. Fig. (44) shows a result on dataset 2 with manual delineations.
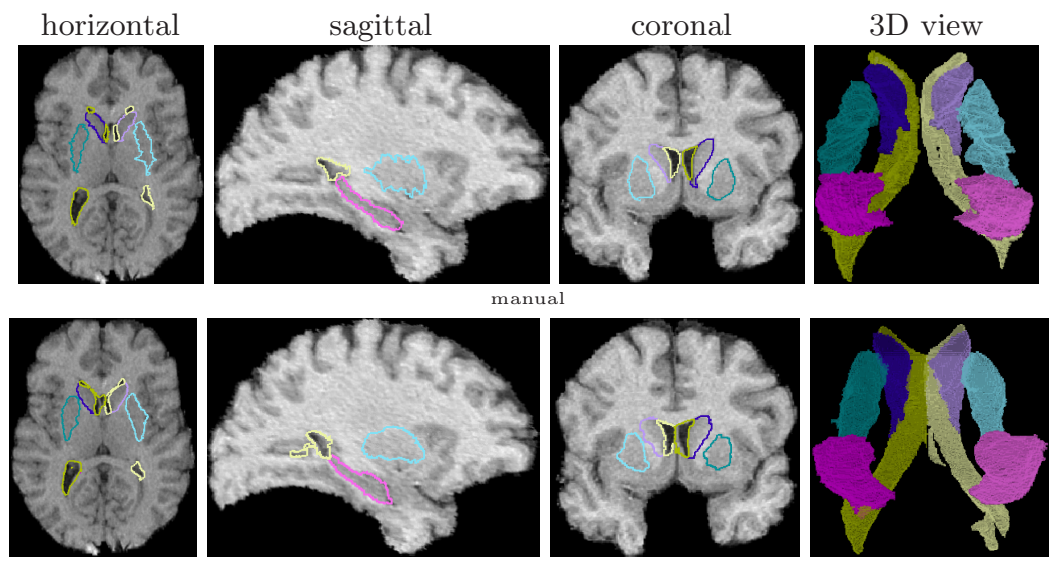

Fig. 5. Results on a typical testing volume. Three planes are shown overlayed with the boundaries of the segmented anatomical structures. The first row shows results manually labeled by an expert. The second row displays the result in [1]. The second row shows the result by hybrid model [1]+3D Canny. The third row shows the result by hybrid model [1]+learned edges. The fourth row displays the result by the new algorithm. The last row shows the result by FreeSurfer [2]. 

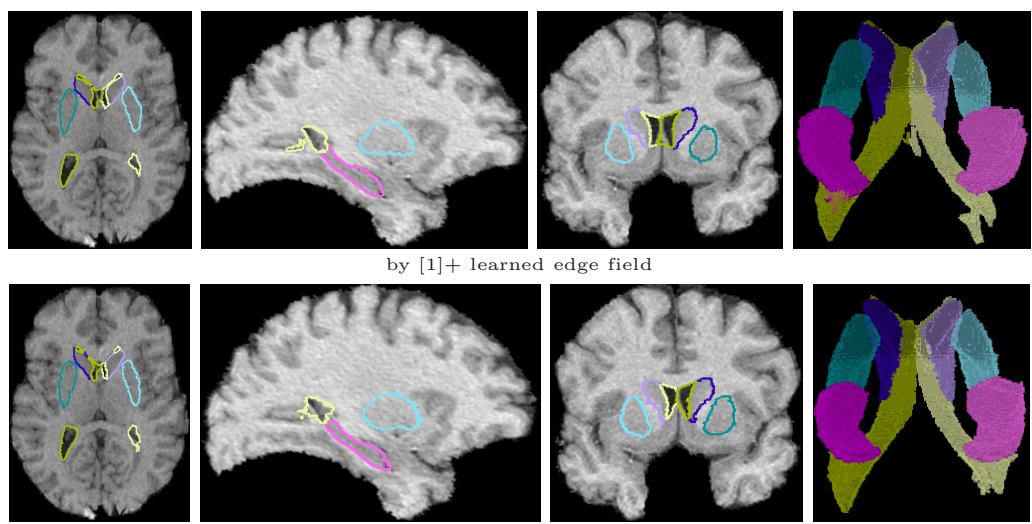

by the method reported in this paper
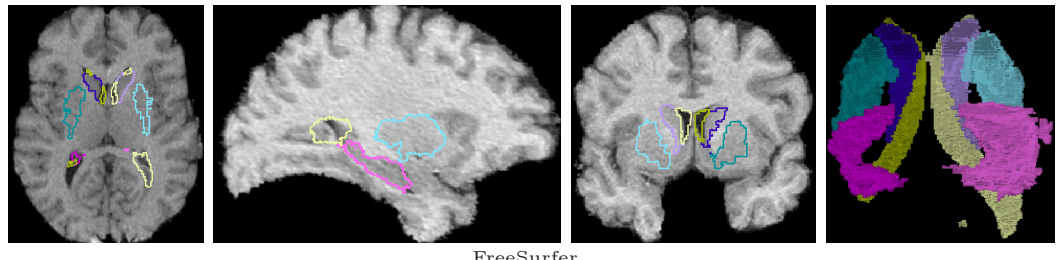

Fig. 5. (continued)

\section{Conclusions}

In this paper, a system for brain anatomical structure segmentation is proposed. The algorithm is very general, and easy to train and test. It has nearly no parameter to tune (a couple very general ones are specified, e.g. the number of weak classifiers for each boosting node). The system makes use of the training data annotated by experts and learns the rules implicitly from examples. We are in the process of getting more $(50 \sim 100)$ structures manually annotated for training our algorithm. Our goal is to allow the system to eventually perform full brain segmentation, and the algorithm reported in this paper facilitates this. Also, more thorough experiments compared with other existing algorithms, e.g. [9], will be conducted.

\section{Acknowledgment}

This work was funded by the NIH through the NIH Roadmap for Medical Research, Grant U54 RR021813 entitled Center for Computational Biology (CCB).

\section{References}

1. Tu, Z., Narr, C., Dollar, P., Thompson, P., Toga, A.: Brain Anatomical Structure Parsing by Hybrid Discriminative/Generative Models. review of IEEE Trans. on Medical Imaging 
2. Fischl, B., Salat, D.H., Busa, E., Albert, M., Dieterich, M., Haselgrove, C., van der Kouwe, A., Killiany, R., Kennedy, D., Klaveness, S., Montillo, A., Makris, N., Rosen, B., Dale, A.M.: Whole brain segmentation: automated labeling of neuroanatomical structures in the human brain. Neuron 33, 341-355 (2002)

3. Freund, Y., Schapire, R.E.: A decision-theoretic generalization of on-line learning and an application to boosting. J. of Comp. and Sys. Sci. 55(1) (1997)

4. Friedman, J., Hastie, T., Tibshirani, R.: Additive logistic regression: a statistical view of boosting, Dept. of Statistics, Stanford Univ. Technical Report (1998)

5. Kass, M., Witkin, A., Terzopoulos, D.: Snakes: Active Contour Models. Int'l. J. Computer Vision 1(4), 321-332 (1988)

6. Lao, Z., Shen, D., Jawad, A., Karacali, B., Liu, D., Melhem, E., Bryan, N., Davatzikos, C.: Automated Segmentation of White Matter Lesions in 3D Brain MR Images, Using Multivariate Pattern Classification. In: Proc. of 3rd IEEE In'l Symp. on Biomedical Imaging, Arlington, VA, USA, April 6-9, IEEE Computer Society Press, Los Alamitos (2006)

7. Monga, O., Deriche, R., Malandain, G., Cocquerez, J.P.: Recursive filtering and edge closing: Two primary tools for 3D edge detection. Image and Vision Computing 9(4), 203-214 (1991)

8. Rohlfing, T., Russakoff, D.B., Maurer Jr., C.R.: Performance-based classifier combination in atlas-based image segmentation using expectation-maximization parameter estimation. IEEE Trans. on Medical Imaging 23(8) (2004)

9. Pohl, K.M., Fisher, J., Kikinis, R., Grimson, W.E.L., Wells, W.M.: A Bayesian model for joint segmentation and registration. NeuroImage 31, 228-239 (2006)

10. Quinlan, J.R.: Improved use of continuous attributes in C4.5. J. of Art. Intell. Res. 4, 77-90 (1996)

11. Tu, Z.: Probabilistic boosting tree: Learning discriminative models for classification, recognition, and clustering. In: Proc. of ICCV (2005)

12. Woods, R.P., Mazziotta, J.C., Cherry, S.R.: MRI-PET registration with automated algorithm. Journal of Computer Assisted Tomography 17, 536-546 (1993)

13. Yang, J., Staib, L.H., Duncan, J.S.: Neighbor-Constrained Segmentation with Level Set Based 3D Deformable Models. IEEE Trans. on Medical Imaging 23(8) (2004) 